\title{
High density genetic maps of St. Augustinegrass and applications to comparative genomic analysis and QTL mapping for turf quality traits
}

\author{
Xingwang $\mathrm{Yu}^{1 *} \mathrm{D}$, Jennifer A. Kimball ${ }^{1,2}$ and Susana R. Milla-Lewis ${ }^{1}$
}

\begin{abstract}
Background: St. Augustinegrass [Stenotaphrum secundatum (Walt.) Kuntze] is a warm-season, perennial turfgrass species well adapted for home lawns and commercial landscapes with economic and ecological value. However, a lack of genomic resources in St. Augustinegrass has hindered the full utilization of genetic variance for maximizing genetic gain and limited our understanding of the species' evolution.

Results: In this study, we constructed the first high-density linkage map for St. Augustinegrass using a genotyping by sequencing (GBS) approach. The integrated linkage map consists of 2871 single nucleotide polymorphism (SNP) and 81 simple sequence repeat (SSR) markers, spanning $1241.7 \mathrm{cM}$, with an average distance of $0.4 \mathrm{cM}$ between markers, and thus represents the densest genetic map for St. Augustinegrass to date. Comparative genomic analysis revealed interchromosome arrangements and independent nested chromosome fusion events that occurred after St. Augustinegrass, foxtail millet, sorghum, and rice diverged from a common ancestor. Forty-eight candidate quantitative trait loci (QTL) were detected for turf quality-related traits, including overall turf quality, leaf texture, genetic color, and turf density. Three hot spot regions were identified on linkage groups LG3 and LG8, where multi-QTL for different traits overlapped. Several leaf development related genes were contained within these identified QTL regions.
\end{abstract}

Conclusions: This study developed the first high-density genetic map and identified putative QTL related to turf quality, which provide valuable genetic resources for marker-assisted selection (MAS) in St. Augustinegrass.

Keywords: St. Augustinegrass, Linkage map, Comparative genomic analysis, QTL

\section{Background}

St. Augustinegrass (Stenotaphrum secundatum [Walt.] Kuntze) is a warm-season turfgrass that is well adapted to tropical and subtropical regions of the world [1]. The grass is native to the Gulf of Mexico, the West Indies and Western Africa, and has been widely used along the Gulf Coast in the U.S., Southern Mexico, throughout the Caribbean region, South America, South Africa, Western Africa, Australia, and the South Pacific and Hawaiian Islands. St. Augustinegrass exhibits superior shade tolerance and moderately low input requirements compared

\footnotetext{
*Correspondence: xyu15@ncsu.edu

'Department of Crop and Soil Sciences, N.C. State University, Box 7620, Raleigh, NC 27695-7620, USA

Full list of author information is available at the end of the article
}

to other turf species [2]. It has been a popular turfgrass in the southern United States for its broad leaf blades, and rapid stolon elongation, which makes the grass well-suited for sod production [3].

St. Augustinegrass belongs to tribe Paniceae in the subfamily Panicoideae, one of the largest subfamilies in Poaceae (grass family). This large subfamily contains many species of important economic value, including lawn grasses centipedegrass (Eremochloa ophiuroides) and St. Augustinegrass (Stenotaphrum secundatum) [4], biofuel stocks switchgrass (Panicum virgatum) [5] and other important crops, such as foxtail millet (Setaria italica), sorghum (Sorghum bicolor) and corn (Zea mays) [6]. This subfamily includes enormous morphological, physiological and cytological diversity and several basic

(c) The Author(s). 2018 Open Access This article is distributed under the terms of the Creative Commons Attribution 4.0 International License (http://creativecommons.org/licenses/by/4.0/), which permits unrestricted use, distribution, and reproduction in any medium, provided you give appropriate credit to the original author(s) and the source, provide a link to the Creative Commons license, and indicate if changes were made. The Creative Commons Public Domain Dedication waiver (http://creativecommons.org/publicdomain/zero/1.0/) applies to the data made available in this article, unless otherwise stated. 
chromosome numbers. It has been reported that $x=9$ and $x=10$ predominate the basic chromosome number in Panicoideae [6]. Understanding the mechanism by which chromosome numbers evolved is a key component to successfully deciphering genome evolution in the grasses. Thus, there is great interest in understanding the comparative genomics relationships among St. Augustinegrass and other members within Panicoideae.

The basic chromosome number of St. Augustinegrass is $x=9$, with diploids $(2 n=2 x=18)$, triploids $(2 n=3 x=27)$, and tetraploids $(2 n=4 x=36)$ reported [7], although aneuploid $(2 n=28,2 n=32)$ genotypes have also been identified more recently [3]. Molecular markers can be used to identify cultivars and characterize germplasm diversity. More importantly, molecular markers can be used to construct genetic maps, and together with phenotypic data, can be used to map genomic regions controlling traits of interest. In past years, progress has been made in the development of molecular markers and construction of linkage maps in St. Augustinegrass. Simple sequence repeats (SSR) markers have been developed and used to evaluate St. Augustinegrass [8, 9]. Recently, Kimball et al. reported the first linkage map for St. Augustinegrass with 160 SSR markers consisting of 9 linkage groups and spanning $1176.24 \mathrm{cM}$ [10]. In addition, multiple QTL associated with winter survival-related traits were identified using this linkage map. However, this is a low marker density map, making it insufficient for fine mapping traits of interest. Furthermore, PCR-based markers like SSRs make the genotyping time and labor required high. In order to conduct large-scale association mapping and improve the efficiency of marker-assisted selection (MAS) in breeding efforts, the development of high-density linkage maps using new types of molecular markers such as single nucleotide polymorphism (SNP) is essential.

High-throughput sequencing technologies provide new tools for developing large numbers of SNP markers, exploring species diversity, constructing linkage maps and performing genome-wide association studies (GWAS) [11, 12]. Genotyping by sequencing (GBS), a simple highly multiplexed system for constructing reduced representation libraries for genetic analysis and genotyping, is becoming increasingly important as a cost effective and unique tool for MAS breeding in a range of plant species [13-17]. Most importantly, GBS is an excellent approach for plant breeding applications even in the absence of a reference genome [13].

High density linkage maps are valuable genetic tools for mapping quantitative trait loci (QTL) and map-based gene cloning. To fully explore the genomic evolution of St. Augustinegrass and understand the genetic mechanisms that determine turf quality, in this study we aimed to 1 ) construct high density linkage maps integrating
SNP and SSR markers using a GBS approach; 2) conduct a comparative genomic study with other grass species and 3) identify QTL associated with turf quality traits.

\section{Results}

Genotype by sequencing and SNP discovery

The "pseudo-F2" mapping strategy enabled us to generate linkage maps for non-inbred species [18]. GBS libraries were constructed for the two parents ('Raleigh' and 'Seville') and 115 pesudo- $F_{2}$ hybrids and sequenced on Illumina HiSeq 2500. A total of 236.6 million raw singleend reads were obtained from sequencing. By identifying barcodes and cut sites, low quality reads were removed and 211.5 million high quality reads were kept. The number of reads for each hybrid ranged from 0.48 million to 3.3 million, with an average of 1.6 million. The parental lines were sequenced at a relatively higher depth than the $F_{1}$ hybrids to maximize the potential of detecting segregating SNPs in the parents. A total of 7.6 million and 6.5 million reads were obtained for 'Raleigh' and 'Seville', respectively, approximately 4.7 and 4 fold the hybrids averages (Additional file 1: Table S2).

The non-reference GBS SNP calling pipeline UNEAK was used to discover SNP markers. A total of 19,810 bi-allelic polymorphic sites were identified between the two parents. After discarding SNPs with low genotyping rates $(<90 \%)$ and significantly distorted $(P<0.01)$ segregation ratios, 2871 high quality SNPs were obtained for linkage map construction. These consisted of 1100 ' $\mathrm{lm} x$ ll', 1571 'nn x np', and 200 'hk x hk' type SNPs (For definition of allele scoring refer to Methods section.).

\section{Linkage map construction}

Filtered markers were loaded into JoinMap 4.0 [19] for linkage map construction. Initially, ' $\mathrm{lm} \times \mathrm{ll}$ ' and ' $\mathrm{nn} \mathrm{x} \mathrm{np}$ ' type SNPs were used to construct separate linkage maps for each parent. For the linkage map of 'Raleigh', 1100 SNPs were mapped into nine linkage groups (LGs) (Fig. 1a), named RLG1-RLG9, which were designated based on homology between the 'Raleigh' map and the foxtail millet genome. The number of SNP markers on each LG varied with a maximum of 151 on RLG7 and a minimum of 78 on RLG4 (Table 1). All nine LGs spanned a total distance of $1238.7 \mathrm{cM}$, with an average distance of $1.1 \mathrm{cM}$ between markers. While RLG3 was the longest with $197.2 \mathrm{cM}$, RLG4 was the shortest with $90.0 \mathrm{cM}$. The same LG number was observed on the linkage map of 'Seville', named SLG1-SLG9 based on homology with the genome of foxtail millet (Fig. 1b). Total length for all the 'Seville' LGs was $914.2 \mathrm{cM}$, with average distance $0.6 \mathrm{cM}$ between markers (Table 1). For each individual LG, sizes ranged from $82.6 \mathrm{cM}$ of SLG4 to $119.3 \mathrm{cM}$ of SLG5. SLG3 included the most SNPs (223), while SLG6 included least (128). Compared with the 'Raleigh' map, the 


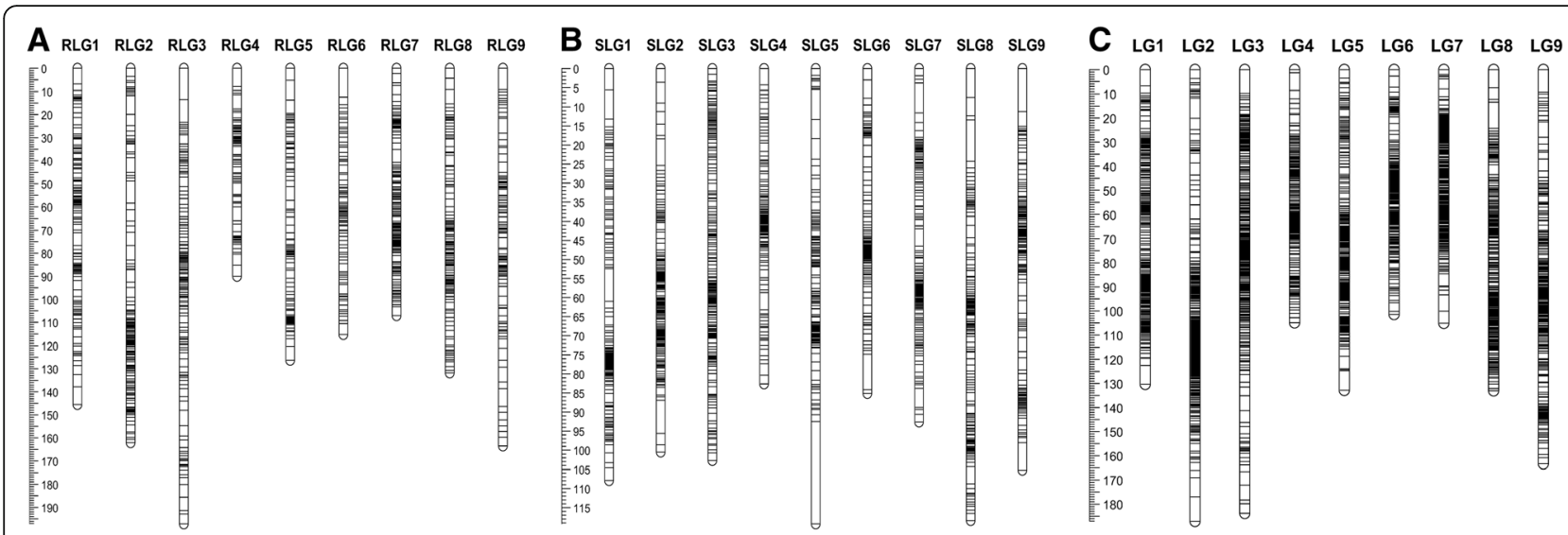

Fig. 1 Distribution of molecular markers on parental and integrated genetic maps of St. Augustinegrass. a Map of parent 'Raleigh'. b Map of parent 'Seville'. c Integrated map from both parents. A black bar indicates a molecular maker. Linkage group number is shown on $x$-axis and genetic distance is shown on $y$-axis (cM)

'Seville' map included more SNP markers but covered a shorter length.

The 'Im $\mathrm{x}$ ll' and 'nn $\mathrm{x}$ np' type together with 'hk $\mathrm{x}$ hk' type SNP and previously identified SSR markers [10] were used to construct an integrated map for St. Augustinegrass. The integrated map consisted of 2871 SNP and 81 SSR markers, which were distributed on nine LGs (Fig. 1c). The total genetic length of the integrated map was $1241.7 \mathrm{cM}$, with an average distance of $0.4 \mathrm{cM}$ between markers (Table 1 ). The longest LG was LG2 $(187.1 \mathrm{cM})$, while LG6 was the shortest one $(101.4 \mathrm{cM})$. The full information of markers in 'Raleigh', 'Seville' and integrated maps can be found in Additional file 1: Tables S3, S4 and S5. By anchoring the SSR markers with the previous linkage map constructed by Kimball et al. [10], the correspondence of LGs and kLGs (Kimball's linkage group) were identified as: LG1-kLG6, LG2-kLG1, LG3-kLG3, LG4-kLG9, LG5-kLG7, LG6-kLG4, LG7-kLG8, LG8-kLG5 and LG9-kLG2. (Table 1 appeared after this paragraph).

\section{Comparative genomics analysis among grasses}

Comparative genomics analysis between St. Augustinegrass and three other model grass species (foxtail millet, sorghum and rice) was performed to investigate syntenic conservation and chromosome rearrangements between them. Both St. Augustinegrass and foxtail millets belong to the Panicoideae subfamily in the grass family, with an equivalent basic chromosome number of $x=9$. The blastn search against the foxtail millet genome revealed both 'Raleigh' and 'Seville' LGs showed orthologous relationships with foxtail millet chromosomes. St. Augustinegrass LGs were numbered in order based on the orthology to foxtail millet chromosomes. Among 1100 SNPs on 'Raleigh' LGs, 603 SNPs (54.8\%) could be positioned on foxtail millet chromosomes, 544 of which could be mapped on the orthologous chromosomes (Table 2 and Additional file 1: Table S6). Meanwhile, 873 (55.6\%) SNPs mapped on 'Seville' LGs were located on foxtail millet chromosomes, 794 of which could be placed on orthologous chromosomes (Table 2 and

Table 1 Linkage group (LG) assignment, marker numbers, LG size and marker density in St. Augustinegrass SNP-based genetic maps

\begin{tabular}{|c|c|c|c|c|c|c|c|c|c|}
\hline \multirow[b]{2}{*}{ Linkage group } & \multicolumn{3}{|c|}{ Number of markers } & \multicolumn{3}{|l|}{ Size $(c M)$} & \multicolumn{3}{|c|}{ Average distance between markers (cM) } \\
\hline & 'Raleigh' map & 'Seville' map & Integrated map & 'Raleigh' map & 'Seville' map & Integrated map & 'Raleigh' map & 'Seville' map & Integrated map \\
\hline LG1 & 132 & 172 & $337(5)$ & 145.5 & 107.9 & 130.3 & 1.1 & 0.6 & 0.4 \\
\hline LG2 & 148 & 189 & $373(14)$ & 162.1 & 100.5 & 187.1 & 1.1 & 0.5 & 0.5 \\
\hline LG3 & 142 & 223 & $390(8)$ & 197.2 & 102.7 & 183.8 & 1.4 & 0.5 & 0.5 \\
\hline LG4 & 78 & 150 & $240(5)$ & 90.0 & 82.6 & 104.9 & 1.2 & 0.6 & 0.4 \\
\hline LG5 & 108 & 175 & $326(11)$ & 126.3 & 119.3 & 132.8 & 1.2 & 0.7 & 0.4 \\
\hline LG6 & 93 & 128 & $279(12)$ & 115.3 & 85.1 & 101.4 & 1.2 & 0.7 & 0.4 \\
\hline LG7 & 151 & 200 & $374(3)$ & 107.1 & 92.6 & 105.1 & 0.7 & 0.5 & 0.3 \\
\hline LG8 & 129 & 164 & $328(12)$ & 131.9 & 118.4 & 133.0 & 1.0 & 0.7 & 0.4 \\
\hline LG9 & 119 & 170 & $305(11)$ & 163.5 & 105.2 & 163.3 & 1.4 & 0.6 & 0.5 \\
\hline Total & 1100 & 1571 & $2952(81)$ & 1238.7 & 914.2 & 1241.7 & 1.1 & 0.6 & 0.4 \\
\hline
\end{tabular}

* Number in parentheses indicate number of SSR markers included 
Table 2 Number of St. Augustinegrass markers that mapped to foxtail millet, sorghum and rice genomes

\begin{tabular}{|c|c|c|c|c|c|c|c|c|c|c|}
\hline \multirow[b]{2}{*}{ 'Raleigh' LG } & \multirow[b]{2}{*}{ 'Seville' LG } & \multirow[b]{2}{*}{ Foxtail millet chr. } & \multicolumn{3}{|c|}{$\begin{array}{l}\text { Number of markers mapped } \\
\text { to foxtail millet genome }\end{array}$} & \multicolumn{3}{|c|}{$\begin{array}{l}\text { Number of markers mapped to } \\
\text { sorghum genome }\end{array}$} & \multicolumn{2}{|c|}{$\begin{array}{l}\text { Number of markers mappec } \\
\text { to rice genome }\end{array}$} \\
\hline & & & 'Raleigh' & Seville' & Sorghum chr. & 'Raleigh' & 'Seville' & Rice chr. & 'Raleigh' & 'Seville' \\
\hline RLG1 & SLG1 & Chrl & $75(70)$ & $122(118)$ & Chr4 & $26(24)$ & $51(48)$ & Chr2 & $18(17)$ & $36(32)$ \\
\hline RLG2 & SLG2 & Chrll & $79(76)$ & $103(97)$ & Chr2 & $28(24)$ & $42(40)$ & Chr9 + Chr7 & $17(8+5)$ & $30(21+4)$ \\
\hline RLG3 & SLG3 & Chrlll + ChrVII & $81(65+12)$ & $120(82+25)$ & Chr8 + Chr9 & $20(7+10)$ & $44(11+26)$ & Chr12+Chr5 & $15(7+4)$ & $34(9+19)$ \\
\hline RLG4 & SLG4 & ChrlV & $44(42)$ & $73(66)$ & Chr10 & $18(17)$ & $25(22)$ & Chr6 & $8(8)$ & $10(8)$ \\
\hline RLG5 & SLG5 & ChrV & $65(59)$ & $90(84)$ & Chr3 & $19(18)$ & $38(32)$ & Chr1 & $14(14)$ & $27(22)$ \\
\hline RLG6 & SLG6 & ChrVl & $34(30)$ & $66(54)$ & Chr7 & $10(9)$ & $29(24)$ & Chr8 & $9(5)$ & $22(15)$ \\
\hline RLG7 & SLG7 & ChrVII + Chrlll & $89(67+15)$ & $125(103+18)$ & Chr6 & $33(28)$ & $41(37)$ & Chr4 & $18(14)$ & $24(21)$ \\
\hline RLG8 & SLG8 & ChrVIII & $57(48)$ & $65(45)$ & Chr5 & $20(15)$ & $22(18)$ & Chr11 & $12(7)$ & $15(10)$ \\
\hline RLG9 & SLG9 & ChrlX & $68(60)$ & 109 (102) & Chr1 & $25(20)$ & $46(38)$ & Chr3 + Chr10 & $17(11+4)$ & $22(15+2)$ \\
\hline Total & & & $603(544)$ & 873 (794) & & 199 (172) & 338 (296) & & $128(104)$ & $220(178)$ \\
\hline
\end{tabular}

* Number in parentheses indicates markers that could be mapped to orthologous chromosomes

Additional file 1: Table S7). In addition, dot-plot diagrams showed that there was high collinearity between the genomes of St. Augustinegrass and foxtail millet (Fig. 2a, b).

Despite the high degree of synteny and collinearity between St. Augustinegrass and foxtail millet, several chromosome rearrangements were observed that differentiate the two species. These inter-chromosomal rearrangements occurred between ChrIII and ChrVII to result in RLG3 and RLG7, and SLG3 and SLG7 (Fig. 2c, d). One end of RLG3 as well as SLG3 was orthologous with the end of ChrVII in foxtail millet, while one end of RLG7 and SLG7 was positioned on ChrIII (Fig. 2c, d). In addition, there were

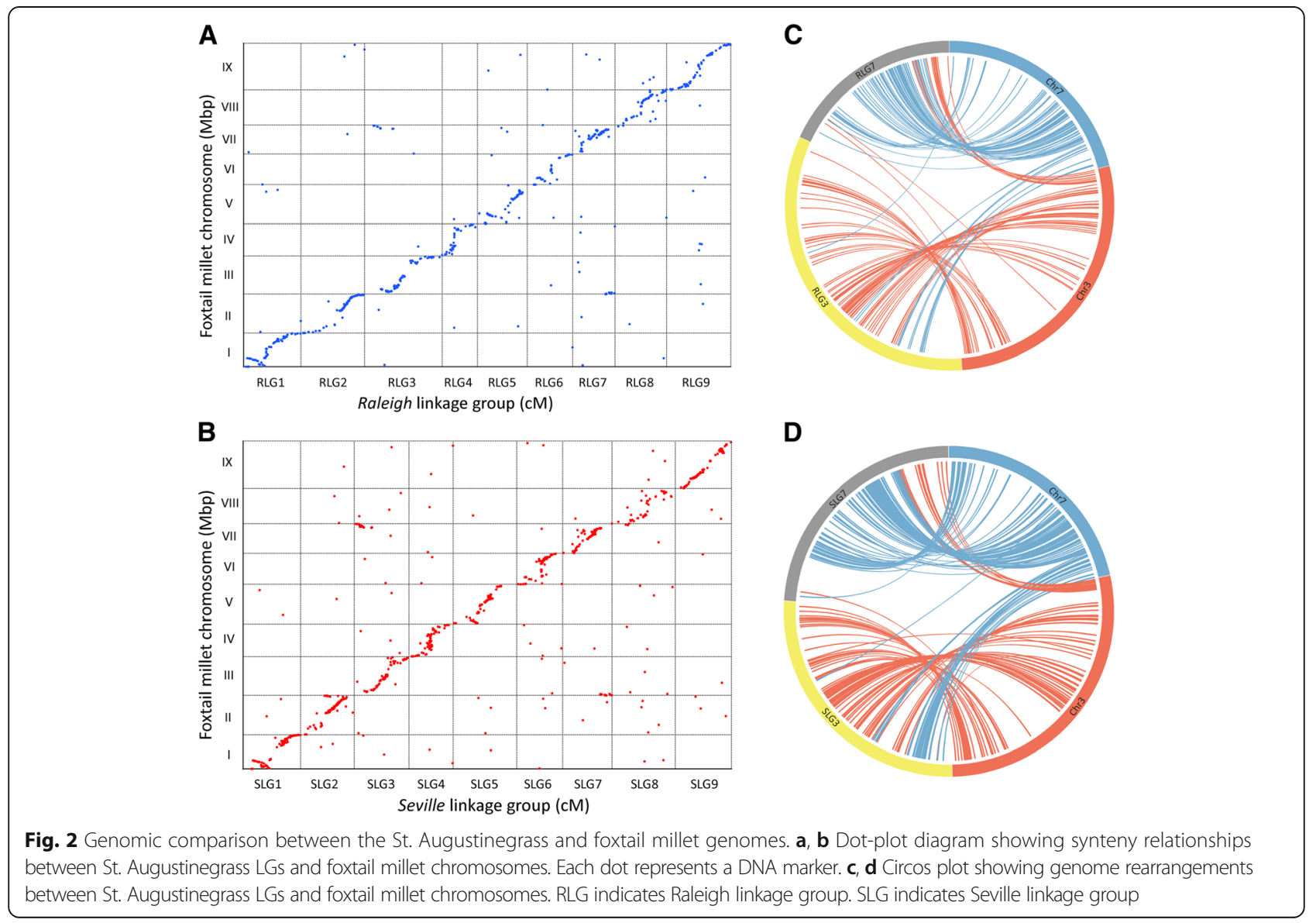


chromosome inversions that occurred near the ends of RLG1, RLG5, RLG6 and homologous SLG1, SLG5, SLG6 (Fig. 2a, b).

Sorghum is another member of the Panicoideae subfamily, but with a higher basic chromosome number than St. Augustinegrass $(x=10)$. Comparative genomics analysis identified 199 and 338 SNPs on 'Raleigh' and 'Seville' LGs that could be located on sorghum chromosomes (Table 2, Additional file 1: Tables S8 and S9). Dot-plots showed high collinearity between genomes of St. Augustinegrass and sorghum (Fig. 3a, b). There was one to one correspondence for the orthologous chromosomes between St. Augustinegrass and sorghum, except that $\mathrm{R}(\mathrm{S}) \mathrm{LG} 3$ were orthologous with both $\mathrm{Chr} 8$ and $\mathrm{Chr} 9$ in sorghum (Fig. 3c). This relationship indicated that a nest chromosome fusion event occurred between Chr8 and Chr9 in sorghum to form R(S)LG3 in St. Augustinegrass.

The rice genome (Oryza sativa) has been commonly used as reference comparison for genome analysis in the grass family as it has retained 12 basic chromosomes from the common ancestor of grass. There were 128 and 220 SNPs on 'Raleigh' and 'Seville' LGs that could be mapped on rice chromosomes (Table 2, Additional file 1: Tables S10 and S11). R(S)LG2, R(S)LG3 and R(S)LG9 were orthologous with rice Chr7 and Chr9, Chr12 and Chr5, Chr3 and Chr10, respectively. Rice Chr9, Chr5 and Chr10 were fused to the middle region of Chr7, Chr12 and Chr3 to form R(S)LG2, R(S)LG3 and R(S)LG9, respectively (Fig. $4 c, d)$. These results suggested that three separate pairs of chromosomes fused to form three chromosomes in St. Augustinegrass during the evolution of the grass family. (Table 2 appeared after this paragraph).

\section{QTL identification for turf quality-related traits}

Four turf quality-related traits (overall turf quality, leaf texture, genetic color, and turf density) evaluated under multiple environments in Kimball et al. were selected for QTL analysis [10]. A total of 48 candidate QTL were detected in all environments (Table 3). Among them, 11 QTL were identified for overall turf quality, 11 QTL for leaf texture, 16 QTL for genetic color and 10 QTL for turf density. All 48 QTL were distributed on all linkage groups with the exception of LG6 and LG7.

Several QTL were identified repetitively in different environments. For overall turf quality, position $38.27 \mathrm{cM}$ on LG3 was confirmed in LW2013, LS2013 and Across, while $53.57 \mathrm{cM}$ on LG3 was detected both in LW2013 and Across (Table 3). Another overlap region for overall turf quality was identified on 40.44 cM of LG8 in LS2013, LW2015 and Across. For genetic color, the 41.44-42.44 cM interval on LG8 was repetitively confirmed on all
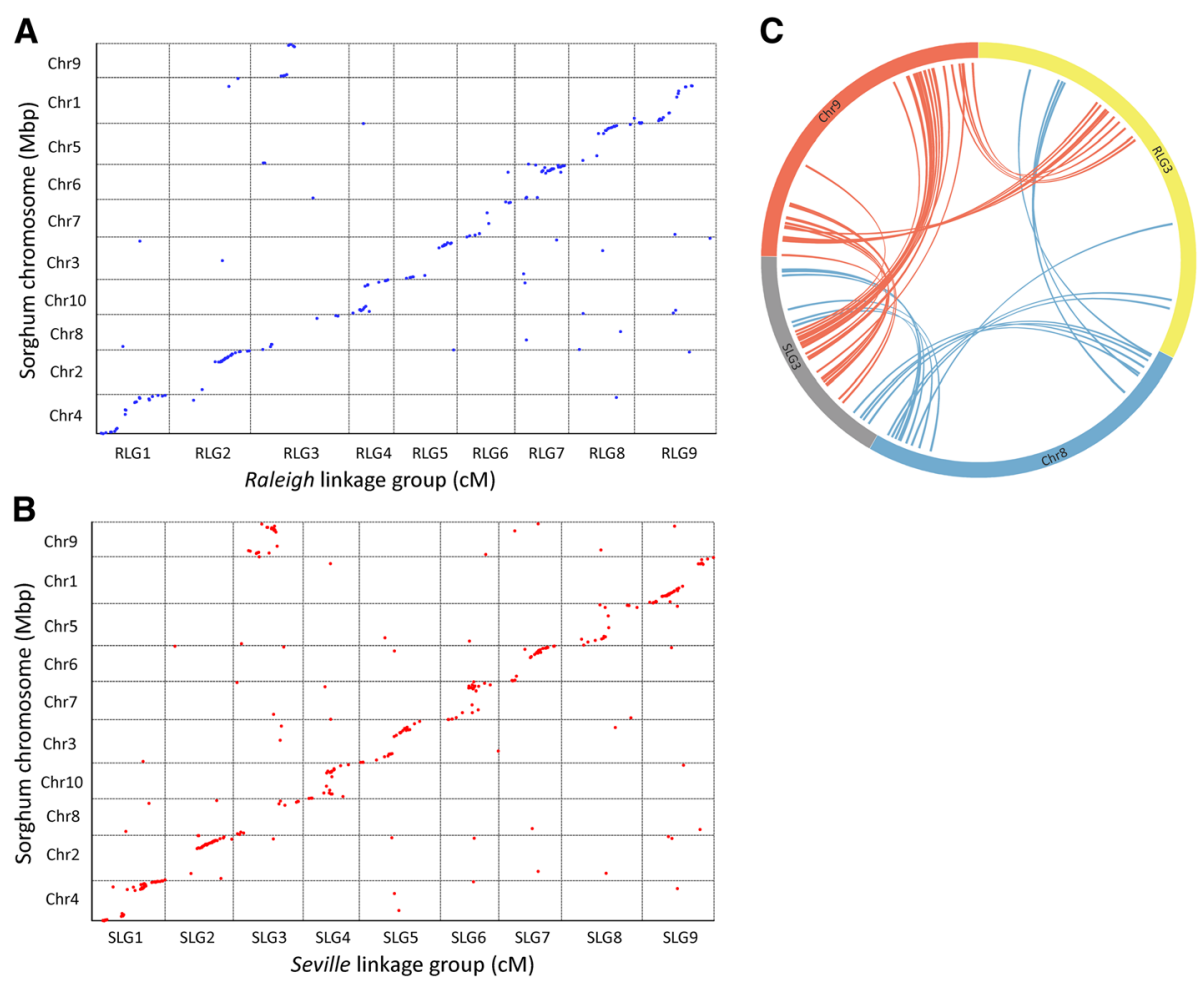

Fig. 3 Genomic comparison between the St. Augustinegrass and sorghum genomes. a, b Dot-plot diagram showing synteny relationships between St. Augustinegrass LGs and sorghum chromosomes. Each dot represents a DNA marker. c Circos plot showing genome rearrangements between St. Augustinegrass LGs and sorghum chromosomes. RLG indicates Raleigh linkage group. SLG indicates Seville linkage group 

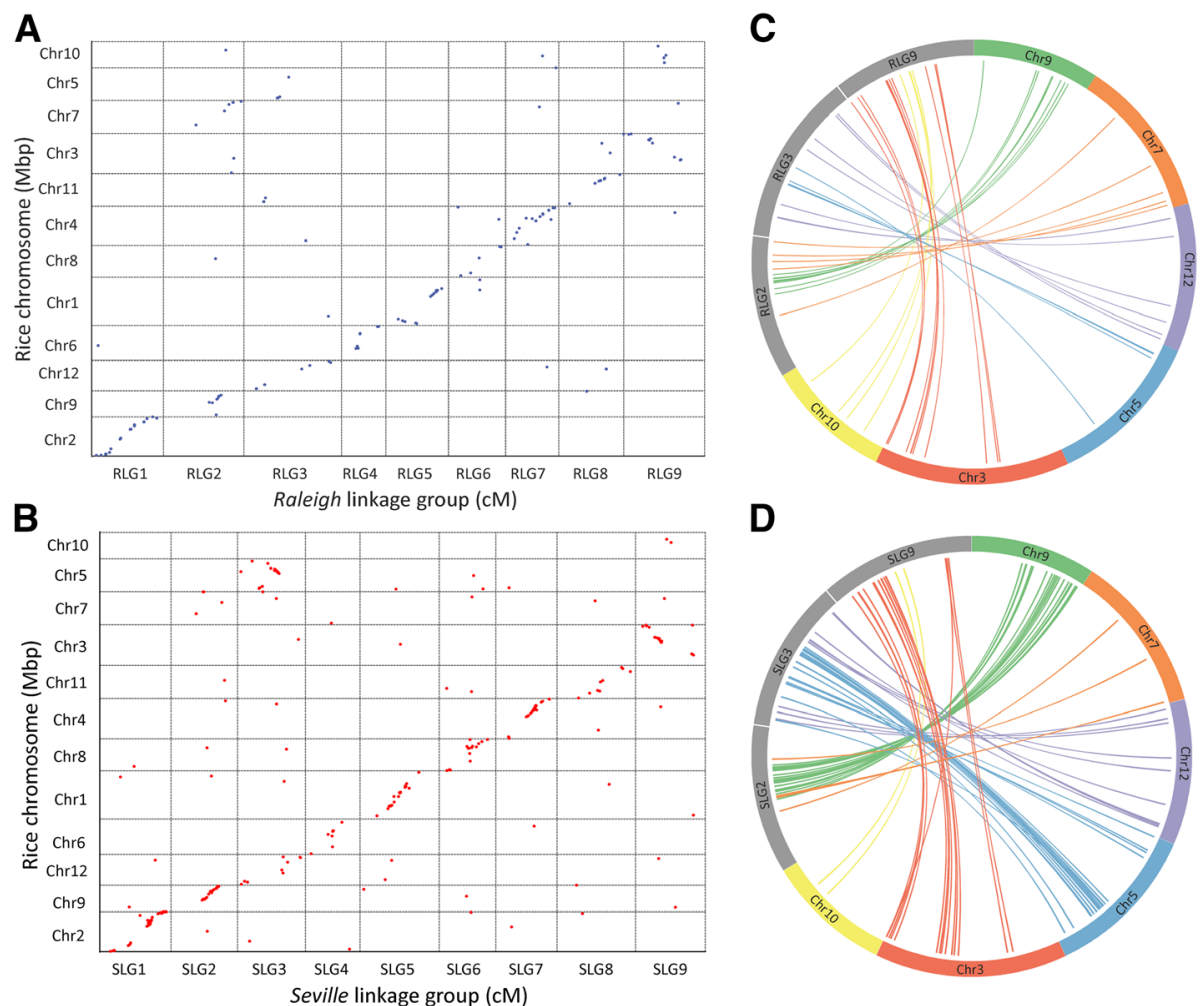

Fig. 4 Genomic comparison between the St. Augustinegrass and rice genomes. a, b Dot-plot diagram showing synteny relationships between St. Augustinegrass LGs and rice chromosomes. Each dot represents a DNA marker. c, d Circos plot showing chromosome fusion between St. Augustinegrass LGs and rice chromosomes. RLG indicates Raleigh linkage group. SLG indicates Seville linkage group.

environments, while the $81.61-82.01 \mathrm{cM}$ interval on LG5 was detected both in LW2013 and Across (Table 3). Four overlapping regions were identified for turf density, including $53.57 \mathrm{cM}$ and $37.55-40.15 \mathrm{cM}$ on LG3, 45.22$56.52 \mathrm{cM}$ on LG4 and $38.08 \mathrm{cM}$ on LG8 (Table 3). For leaf texture, three overlapping regions were found on LG3 (38.27 $\mathrm{cM}$ and 54.94 cM) and LG5 (90.54 cM) (Table 3).

In addition, we found QTL for different traits that co-located to the same region, especially on LG3 and LG8. There were seven QTL that overlapped in the interval of $35.66-40.15 \mathrm{cM}$ on LG3, which included overall turf quality, turf density and leaf texture. On the same linkage group, there was another hot spot region $(47.03-54.93 \mathrm{cM})$ that contained seven QTL for overall turf quality, turf density and leaf texture (Table 3). On LG8, 10 QTL for overall turf quality, turf density and genetic color overlapped in the $38.08-50.27 \mathrm{cM}$ region (Table 3).

Sequences of markers within QTL regions were subsequently used for gene annotation analysis. The results showed several genes related to leaf formation and development, including: leaf trichome morphogenesis, anthocyanin biosynthetic, leaf senescence, auxin biosynthesis, cell wall metabolism, and wax/lipid biosynthesis (Additional file 1: Table S12) were included in these regions. The gene ontology (GO) analysis of these genes suggested a possible association between these genes and turf leaf morphology related traits. (Table 3 appeared after this paragraph).

\section{Discussion}

\section{High density genetic map for St. Augustinegrass}

Breeding efforts have been made to improve turf quality and its tolerance to biotic and abiotic stresses. Although conventional breeding methods are used in most turfgrass breeding programs, molecular breeding methods such MAS are becoming increasingly popular [17, 20]. MAS relies on marker-trait associations, hence high density genetic maps containing an abundance of molecular markers will maximize our ability to detect these associations. The St. Augustinegrass linkage map previously generated by Kimball et al. consisted of only 160 SSR markers with an average distance of $8.2 \mathrm{cM}$ between markers [10]. In this study, we constructed an integrated linkage map containing 2871 SNP and 81 SSR markers with nine linkage groups. The map spanned $1241.7 \mathrm{cM}$, with an average distance of $0.4 \mathrm{cM}$ between markers (Table 1). This map highly improved marker density and thus represents the densest genetic map for St. Augustinegrass to date. Furthermore, our map also integrates 
Table 3 Number and characteristics of QTL identified for turf quality traits under both individual and across environments

\begin{tabular}{|c|c|c|c|c|c|c|c|c|c|c|}
\hline Trait $^{a}$ & Environment ${ }^{\mathrm{b}}$ & $\mathrm{LG}$ & $\begin{array}{l}\text { Peak position } \\
\text { (cM) }\end{array}$ & $\begin{array}{l}\text { Interval } \\
(\mathrm{cM})^{c}\end{array}$ & Nearest marker & $\operatorname{LOD}^{d}$ & $\begin{array}{l}\text { Explained variance } \\
(\%)^{\mathrm{e}}\end{array}$ & $A_{f}^{f}$ & $A_{m}^{f}$ & $D^{f}$ \\
\hline \multirow[t]{4}{*}{ LT } & \multirow[t]{4}{*}{ Across } & LG2 & 174.09 & $173.09-176.09$ & SSR16939 & $4.22(3.90)$ & 16.50 & 0.1401 & 0.2081 & 0.1172 \\
\hline & & LG3 & 38.27 & 38.27 & SSR3677 & $4.66(3.90)$ & 18.00 & -0.2001 & 0.0028 & 0.1264 \\
\hline & & LG3 & 54.93 & 54.93 & SNP33683 & $3.94(3.90)$ & 15.50 & -0.0540 & -0.2100 & 0.0819 \\
\hline & & LG5 & 90.54 & 90.54 & SSR14922 & $4.49(3.90)$ & 17.40 & 0.1776 & 0.1610 & 0.1160 \\
\hline LT & LW2013 & LG8 & 52.79 & 52.79 & SSR4549 & $4.86(3.90)$ & 18.70 & -0.1607 & -0.2487 & 0.0118 \\
\hline \multirow[t]{5}{*}{ LT } & \multirow[t]{5}{*}{ LS2013 } & LG2 & 80.90 & $72.61-80.90$ & SSR2502 & $4.25(3.90)$ & 16.60 & 1.3367 & 1.1968 & -1.1999 \\
\hline & & LG2 & 122.33 & $111.13-131.80$ & SNP41141 & $4.45(3.90)$ & 17.30 & 0.2548 & 0.0976 & -0.1621 \\
\hline & & LG2 & 162.83 & 162.83 & SNP8925 & $4.12(3.90)$ & 16.10 & -0.0877 & 0.3335 & -0.0271 \\
\hline & & LG3 & 38.27 & 38.27 & SSR3677 & $4.61(3.90)$ & 17.90 & -0.2474 & 0.0275 & 0.1660 \\
\hline & & LG5 & 90.54 & 90.54 & SSR14922 & $4.49(3.90)$ & 17.40 & 1.2973 & 1.1994 & -1.2529 \\
\hline LT & LW2015 & LG3 & 47.03 & $47.03-54.94$ & SNP49855 & 3.98 (3.90) & 15.60 & -0.0363 & -0.2124 & 0.1925 \\
\hline \multirow[t]{3}{*}{ TQ } & \multirow[t]{3}{*}{ Across } & LG3 & 38.27 & $37.55-40.55$ & SSR3677 & $5.27(3.80)$ & 20.10 & -0.2451 & -0.0453 & 0.1486 \\
\hline & & LG3 & 53.57 & 53.57 & SSR27743 & $5.60(3.80)$ & 21.20 & -0.2276 & -0.0420 & 0.2049 \\
\hline & & LG8 & 40.44 & $37.26-42.44$ & SNP48740 & $6.14(3.80)$ & 23.00 & -0.3092 & -0.2039 & 0.0069 \\
\hline \multirow[t]{3}{*}{ TQ } & \multirow[t]{3}{*}{ LW2013 } & LG3 & 38.27 & 38.27 & SSR3677 & $3.83(3.80)$ & 15.10 & -0.2217 & 0.0234 & 0.1623 \\
\hline & & LG3 & 53.57 & 53.57 & SSR27743 & $4.24(3.80)$ & 16.50 & -0.2159 & 0.0178 & 0.2231 \\
\hline & & LG3 & 79.76 & 79.76 & SSR4343 & $4.66(3.80)$ & 18.00 & -0.3087 & -0.0263 & -0.1565 \\
\hline \multirow[t]{2}{*}{ TQ } & \multirow[t]{2}{*}{ LS2013 } & LG3 & 38.12 & $35.66-38.97$ & SNP41416 & $5.07(3.80)$ & 19.40 & -0.2549 & -0.0424 & 0.2803 \\
\hline & & LG8 & 39.44 & $38.08-40.44$ & SNP48740 & $4.34(3.80)$ & 16.90 & -0.3661 & -0.1268 & 0.0909 \\
\hline \multirow[t]{3}{*}{ TQ } & \multirow[t]{3}{*}{ LW2015 } & LG8 & 40.44 & $36.71-45.4$ & SNP48740 & $5.76(3.90)$ & 21.80 & -0.3047 & -0.2754 & -0.0340 \\
\hline & & LG8 & 49.92 & $48.04-50.27$ & SNP15495 & $4.62(3.90)$ & 17.90 & -0.1179 & -0.2903 & -0.0439 \\
\hline & & LG8 & 53.79 & $53.80-53.83$ & SSR4549 & $4.11(3.90)$ & 16.10 & -0.1321 & -0.3470 & -0.0941 \\
\hline \multirow[t]{3}{*}{ GC } & \multirow[t]{3}{*}{ Across } & LG5 & 74.82 & $74.24-74.82$ & SNP54683 & $4.58(3.90)$ & 17.70 & 0.2695 & -0.0548 & 0.1544 \\
\hline & & LG5 & 81.83 & $81.61-82.01$ & SNP35732 & $4.79(3.90)$ & 18.50 & 0.2814 & -0.0202 & 0.2095 \\
\hline & & LG8 & 41.44 & $36.13-50.27$ & SNP48740 & $6.23(3.90)$ & 23.30 & -0.2638 & -0.2916 & -0.0944 \\
\hline \multirow[t]{3}{*}{ GC } & \multirow[t]{3}{*}{ LW2013 } & LG1 & 102.07 & $100.40-102.08$ & SNP19531 & $4.30(3.90)$ & 16.80 & 0.0070 & -0.3357 & 0.2061 \\
\hline & & LG5 & 81.89 & 74.82-82.09 & SNP61660 & $4.35(3.90)$ & 16.90 & 0.3767 & 0.0233 & 0.1659 \\
\hline & & LG8 & 42.44 & $41.44-42.44$ & SNP27553 & $4.01(3.90)$ & 15.70 & -0.2456 & -0.3268 & -0.1035 \\
\hline \multirow[t]{8}{*}{ GC } & \multirow[t]{8}{*}{ LS2013 } & LG2 & 167.00 & $165.83-170.09$ & SSR17849 & $4.78(4.00)$ & 18.40 & -0.2383 & 0.1909 & 0.0964 \\
\hline & & LG2 & 104.65 & 104.65 & SNP32405 & $4.03(4.00)$ & 15.80 & 0.0887 & -0.1759 & 0.1056 \\
\hline & & LG3 & 68.23 & $68.23-68.24$ & SNP47138 & $4.13(4.00)$ & 16.10 & -0.0678 & -0.2228 & -0.0162 \\
\hline & & LG3 & 75.23 & $75.23-76.33$ & SNP57077 & $4.69(4.00)$ & 18.10 & 0.1176 & -0.2174 & 0.1139 \\
\hline & & LG3 & 87.80 & 87.80 & SNP32773 & $6.64(4.00)$ & 24.70 & -0.1995 & 0.2065 & 0.2110 \\
\hline & & LG5 & 71.77 & $62.44-74.24$ & SNP58680 & $4.59(4.00)$ & 17.80 & 0.2795 & -0.3020 & 0.1802 \\
\hline & & LG8 & 38.08 & $38.08-42.44$ & SSR4381 & $5.12(4.00)$ & 19.60 & -0.1583 & -0.0569 & -0.1734 \\
\hline & & LG9 & 95.48 & $90.12-97.79$ & SNP21678 & $4.88(4.00)$ & 18.80 & 0.1195 & -0.2410 & -0.0226 \\
\hline \multirow[t]{2}{*}{ GC } & \multirow[t]{2}{*}{ LW2015 } & LG1 & 102.07 & $102.07-102.08$ & SNP19531 & $4.09(3.90)$ & 16.00 & -0.0289 & -0.3180 & 0.2213 \\
\hline & & LG8 & 41.44 & $32.50-50.27$ & SNP48740 & $5.64(3.90)$ & 21.40 & -0.3007 & -0.4067 & -0.0704 \\
\hline \multirow[t]{5}{*}{ TD } & \multirow[t]{5}{*}{ Across } & LG3 & 38.27 & $37.55-40.15$ & SSR3677 & $4.94(3.60)$ & 19.00 & -0.3769 & -0.1477 & 0.0900 \\
\hline & & LG3 & 53.57 & 53.57 & SSR27743 & $4.80(3.60)$ & 18.50 & -0.3396 & -0.0607 & 0.2076 \\
\hline & & LG4 & 56.52 & $45.22-56.52$ & SNP5638 & $4.11(3.60)$ & 16.10 & 0.1477 & 0.3091 & 0.0777 \\
\hline & & LG8 & 38.08 & 38.08 & SSR4381 & $4.45(3.60)$ & 17.30 & -0.2515 & -0.1588 & -0.1873 \\
\hline & & LG8 & 56.38 & $56.38-56.62$ & SNP38207 & $4.20(3.60)$ & 16.40 & -0.0725 & -0.2320 & -0.3113 \\
\hline
\end{tabular}


Table 3 Number and characteristics of QTL identified for turf quality traits under both individual and across environments (Continued)

\begin{tabular}{|c|c|c|c|c|c|c|c|c|c|c|}
\hline Trait $^{\mathrm{a}}$ & Environment $^{\mathrm{b}}$ & LG & $\begin{array}{l}\text { Peak position } \\
\text { (cM) }\end{array}$ & $\begin{array}{l}\text { Interval } \\
(\mathrm{cM})^{\mathrm{c}}\end{array}$ & Nearest marker & $\operatorname{LOD}^{\mathrm{d}}$ & $\begin{array}{l}\text { Explained variance } \\
(\%)^{\mathrm{e}}\end{array}$ & $A_{f}^{f}$ & $A_{m}{ }^{f}$ & $D^{f}$ \\
\hline TD & LW2013 & LG3 & 53.57 & 53.57 & SSR27743 & $3.86(3.80)$ & 15.20 & -0.3145 & -0.0217 & 0.2322 \\
\hline \multirow[t]{4}{*}{ TD } & LS2013 & LG3 & 38.39 & $28.80-40.55$ & SNP11022 & $5.82(3.70)$ & 22.00 & -0.3024 & -0.2121 & 0.3327 \\
\hline & & LG3 & 53.57 & 53.57 & SSR27743 & 4.29 (3.70) & 16.70 & -0.3753 & -0.1512 & 0.2056 \\
\hline & & LG4 & 45.84 & $39.32-56.52$ & SNP44515 & $4.23(3.70)$ & 16.50 & 0.2514 & 0.4000 & -0.0119 \\
\hline & & LG8 & 38.31 & $38.08-40.44$ & SNP7787 & 4.69 (3.70) & 18.10 & -0.4282 & -0.2314 & -0.0490 \\
\hline
\end{tabular}

${ }^{\mathrm{a}}$ Turf quality related traits evaluated in this study. LT = leaf texture; TQ = turf quality; GC = genetic color; TD = turf density

${ }^{b}$ From Kimball et al. (2018): LW $2013=$ Raleigh, NC in 2013, LW $2015=$ Raleigh, NC in 2015, LS 2013= Laurel Springs, NC in 2013, Across = combined analysis across environments

${ }^{c}$ Regions with a LOD score above threshold values were considered as potential QTL interval

d Genome-wide LOD threshold in parentheses

e Percentage of phenotypic variation explained by the QTL

${ }^{f}$ Allelic effects were estimated as $A_{f}=\left[\left(\mu_{a c}+\mu_{a d}\right)-\left(\mu_{b c}+\mu_{b d}\right)\right] / 4$ for female (Raleigh) additivity; $A_{m}=\left[\left(\mu_{a c}+\mu_{b c}\right)-\left(\mu_{a d}+\mu_{b d}\right)\right] / 4$ for male $(S e v i l l e)$ additivity and $\mathrm{D}=\left[\left(\mu_{\mathrm{ac}}+\mu_{\mathrm{bd}}\right)-\left(\mu_{\mathrm{ad}}+\mu_{\mathrm{bc}}\right)\right] / 4$ for dominance where $\mu_{\mathrm{ac}}, \mu_{\mathrm{ad}}, \mu_{\mathrm{bc}}$ and $\mu_{\mathrm{bd}}$ are estimated phenotypic means associated to each of the 4 possible genotypic classes ac, bc, ad and bd, deriving for $a n a b \times c d$ cross. Positive value indicates alleles increase the trait value

two types of molecular markers, SNPs and SSRs. While SNP-based high density linkage maps have been successfully used for comparative genomics analysis and QTL mapping in turfgrass [17, 21], SSR markers usually provide high levels of polymorphism information. However, SSRs are more labor intensive while SNP markers are highly abundant and high throughput. Thus, high density genetic maps that include both marker types are advantageous and can be very informative for comparative genomics and QTL analyses. Linkage maps with both SNP and SSR markers have been reported in many species such as pear and wheat [22, 23].

\section{Comparative genomics study}

The grass family is arguably the most important family in agriculture. It provides abundant resources for plant evolutionary studies due to the presence of variation in basic chromosome numbers and a high frequency of polyploidy [24-29]. The advancement of genomic information available for several grass species, such as sorghum, wheat, maize and rice, has promoted numerous comparative genomics studies among grass family members [24-26]. It has been accepted that grass genomes have evolved from a common ancestor which underwent a series of whole-genome duplications, chromosome fusions and rearrangements to produce an intermediate ancestor with 12 basic chromosomes, although there is still argument on the base chromosome number of this common ancestor $(x=5$ or $x=7)$ [27-29]. This presumed 12-chromosomes intermediate ancestor had a very similar chromosome arrangement with current-day rice $(2 n=2 x=24)$. Most grass genomes are hypothesized to have formed from this intermediate ancestor through chromosome fusions, leading to reduction in chromosome numbers and additional rearrangements [29].

Panicoideae has a predominant base chromosome numbers of $x=9$ and $x=10$ [6]. It was previously hypothesized that nested chromosome fusion (NCF) is the dominant mechanism for reduction of chromosome numbers in the grass family $[25,26]$. Panicoideae ancestral genomes with $x=9$ and $x=10$ may have evolved from the $x=12$ intermediate ancestor through three and two, respectively, NCFs. Comparative genomic analysis between sorghum $(x=10)$ and rice $(x=12)$ determined that sorghum chromosome Sb 1 originated from the insertion of the entire rice chromosome Os 10 to the centromeric region of Os 3, while $\mathrm{Sb} 2$ was formed by insertion of Os 9 into Os 7 [25]. In addition, comparison between foxtail millet $(x=9)$ and rice found that foxtail millet chromosomes 2, 3 and 9 were collinear with rice chromosomes 7 and 9, 5 and 12, and 3 and 10 respectively, which indicates that another single NCF occurred in the evolution of the foxtail millet genome in addition to the two NCFs that happened in sorghum [30]. In our study, we found that St. Augustinegrass R(S)LG2, R(S)LG3 and R(S)LG9 were orthologous with rice Chr7 and Chr9, Chr12 and Chr5, Chr3 and Chr10, respectively. The rice Chr9, Chr5 and Chr10 were fused to the middle region of Chr7, Chr12 and Chr3 to form R(S)LG2, R(S)LG3 and R(S)LG9 (Fig. 4). Consistent with previous hypotheses, our results suggest that the St. Augustinegrass genome has evolved from the intermediate ancestor through three NCFs. By comparing the genomes of sorghum and foxtail millet, Zhang et al. found that NCF fused chromosomes 8 and 9 of sorghum in chromosome 3 of foxtail millet [30]. Similar results were observed in the present study, where St. Augustinegrass R(S)LG3 were found to be orthologous with both Chr8 and Chr9 in sorghum (Fig. 3). These results indicated that this NCF event most likely occurred before the divergence of foxtail millet and St. Augustinegrass. The high degree of synteny and collinearity between St. Augustinegrass and foxtail millet observed in our results indicates a very close evolutionary relationship between the two species (Fig. 2a, b). However, there were inter-chromosomal rearrangements 
between St. Augustinegrass LG3 and LG7 and foxtail millet chromosomes 3 and 7 (Fig. 2c, d). Such chromosome rearrangement events might have introduced genetic variation and contributed to divergence between these species.

\section{QTL identification for turf quality related traits}

The high density linkage map generated in this study provided a platform for mapping QTL associated with traits of agronomic importance in St. Augustinegrass. Turfgrass quality is defined as the degree to which a turf conforms to an agreed upon standard. The components of turfgrass quality adopted by NTEP include uniformity, shoot density, leaf texture, leaf orientation, smoothness, and color (NTEP, 2017). In the present study, leaf texture, turf density, genetic color and overall turf quality were selected to evaluate the aesthetic performance of St. Augustinegrass. Kimball et al. detected eight QTL distributed on four LGs for these traits using an SSR-based linkage map [10]. In the present study, 48 putative QTL regions associated with these traits were successfully identified. These QTL regions were distributed on seven of nine LGs (Table 3). The detection power and resolution of QTL mapping was significantly improved by the high density linkage map compared to previous SSR-based map. Among these QTL, a number of occurrences of overlapping QTL for leaf texture, turf density, genetic color and overall turf quality were observed on LG3 (35.66-40.15 cM and $47.03-54.93 \mathrm{cM})$ and LG8 $(38.08-50.27 \mathrm{cM})$ (Table 3). Co-location of QTL for different traits may indicate common genetic mechanisms for these traits, suggesting the importance of these regions for fine mapping as well as MAS [31]. Overlapping of QTL might also indicate that these regions contain genes controlling development and morphology of leaves and shoots. By blasting marker sequences within these regions, several orthologous genes associated with leaf formation and development, including: leaf trichome morphogenesis, anthocyanin biosynthesis, leaf senescence, auxin biosynthesis, cell wall metabolism, and wax/lipid biosynthesis (Additional file 1: Table S12) were found. For example, the orthologous genes of PNH1 in Arabidopsis (PNH/ZLL) and rice (OsPNH1) were both reported to play important roles in the formation of the shoot apical meristem (SAM) from where leaves are produced [32,33]. DTX, which encodes detoxification proteins, also known as Multidrug and Toxic Compound Extrusion (MATE) transporters in plants have been reported to affect plant architecture through the auxin and ABA pathways [34, 35]. It is speculated that the identified QTL regions might be controlling the turf quality traits in part through the orthology of these genes on the genome of St.Augustinegrass. However, further experiments need to be implemented to verify our results and improve the scale and quality of putative QTL, and to identify functional genes controlling turf quality.

Further analysis of the QTL regions found here to be associated with turf quality may help elucidate the genetic mechanisms of these complex traits and improve our ability to select for them during breeding cycles. The high density St. Augustinegrass genetic map, the first of its kind of the species, has the potential to assist in the identification of marker-trait associations for numerous qualitative and quantitative traits of economic and agronomic importance, such as turf quality and tolerance to environmental stresses. These associations can be subsequently used in MAS and thus increase the efficiency of selection in St. Augustinegrass breeding.

\section{Conclusions}

Overall, we identified thousands of SNP markers in St. Augustinegrass using a GBS approach and constructed a high density genetic map including both SNP and SSR markers. To date, this is the most comprehensive genetic map developed for this species. Using this genetic map, we conducted comparative genomics analysis between St. Augustinegrass and foxtail millet, sorghum and rice, which revealed chromosomal rearrangement events that occurred during the evolutionary history of the grass family. These results provide a genetic and genomic basis for future functional gene cloning and genome assembly. In addition, several turf quality-related QTL were identified, which were distributed on different linkage groups. The high density genetic map and identified QTL will enhance turfgrass improvement programs.

\section{Methods \\ Plant materials and DNA extraction}

A pseudo- $\mathrm{F}_{2}$ population consisting of 115 hybrids was derived from a cross between St. Augustinegrass cultivars 'Raleigh' and 'Seville' (both parents are diploids, $2 n$ $=2 x=18$ ) following artificial hybridization methods [10]. This population was obtained from North Carolina State University Center for Turfgrass Environmental Research and Education. Each individual was propagated vegetatively in plastic containers containing Fafard potting mix (Conrad Fafard Inc., Agawam, MA) and maintained in the greenhouse at North Carolina State University, Raleigh, NC, USA. Young leaves of each hybrid along with parents were collected for genomic DNA extraction. The quality of the DNA was first visualized by agarose gel electrophoresis and further tested using a NanoPhotometer (Implen, München, Germany). DNA concentration was quantified using a Hoefer DQ 300 fluorometer (Hoefer, Holliston, United States). 


\section{GBS library construction}

The sequencing library was prepared according to the procedure detailed in Poland et al. with minor modifications [36]. Approximately $200 \mathrm{ng}$ of genomic DNA for each sample (115 hybrids and two parents) was digested with PstI and MspI (New England BioLabs, Inc.; Ipswich, MA) restriction enzymes for $2 \mathrm{~h}$ at $37^{\circ} \mathrm{C}$ in a $20 \mu \mathrm{L}$ volume. The reaction was stopped by incubation at $65^{\circ} \mathrm{C}$ for $20 \mathrm{~min}$. Barcoded adapters (containing unique barcode sequences, details in Additional file 1: Table S1) and a common-Y adapter were ligated to digested genomic DNA fragments at $22^{\circ} \mathrm{C}$ overnight in $40 \mu \mathrm{L}$ volume and stopped at $65^{\circ} \mathrm{C}$ for $20 \mathrm{~min}$. Then, $10 \mu \mathrm{L}$ of each sample was pooled and cleaned up using QIAquick PCR Purification Kit (QIAGEN, Hilden, Germany). After that, purified DNA was amplified using NEB MasterMix (New England BioLabs, Inc.; Ipswich, MA). PCR products were purified and size-selected using GeneRead Size Selection Kit (QIAGEN, Hilden, Germany) to remove adapter dimers and small fragments $(<150 \mathrm{bp})$. The library was size-selected at a range of $250-400 \mathrm{bp}$ using D1000 ScreenTape assay (Agilent, Waldbronn, Germany) and sequenced on Illumina HiSeq 2500 (Illumina, San Diego, United States).

\section{SNP identification and genotyping}

In this study, the non-reference UNEAK pipeline was used to perform SNP discovery and genotyping [15, 37]. GBS raw reads were processed to keep only reads that contained barcodes and the restriction site. High quality reads were trimmed to $64 \mathrm{bp}$ and identical reads were collapsed into tags. Pairwise alignment identified tag pairs with a single base pair mismatch, which could be considered as candidate SNPs. A network filter (Error tolerance rate $=0.03$ ) was employed to discard repeats, paralogs and error tags. The remaining reciprocal tag pairs could then be identified as SNPs. Finally, the SNPs were filtered by sequencing depth $(\geq 10)$, minor allele frequency $(\leq 0.05)$ and call rate $(\geq 90 \%)$ to obtain high quality SNPs.

\section{Linkage map construction}

JoinMap 4.0 [19] was used to construct the linkage map. SNP markers were assigned to three categories according to segregation type: heterozygous in parent 'Raleigh' and homozygous in parent 'Seville' ('lm x ll' type), homozygous in parent 'Raleigh' and heterozygous in parent 'Seville' ('nn $\mathrm{x} n \mathrm{np}^{\prime}$ type), heterozygous in both parents ('hk x hk' type). Markers that showed abnormal segregation ratios (chi-squared test, $\mathrm{df}=2$, cut off value $=9.21$, $P<0.01)$ were excluded from map construction. The ' $\mathrm{lm}$ $\mathrm{x}$ ll' type and 'nn $\mathrm{x} n \mathrm{n}$ ' type SNP markers were used to construct separate parental linkage maps for parent 'Raleigh' and 'Seville', respectively. Meanwhile, 'hk x hk' type SNP markers along with previously identified SSR markers from Kimball et al. [10], were used to integrate the parental linkage groups into a consensus map. All linkage maps were constructed using the regression mapping algorithm with a minimum LOD of 9.0 and a maximum recombination rate of 0.4 (goodness-of-fit jump value 3.0 and ripple value 1). Map distances were calculated using the Kosambi mapping function. The map quality was checked with 'N.N. fit' function in JoinMap 4.0. MapChart 2.32 was used to visualize the linkage maps [38].

\section{Comparative genomics analysis}

The sequences of mapped SNP tags were aligned to genome sequences of model grass species: foxtail millet (Setaria italica), sorghum (Sorghum bicolor) and rice (Oryza sativa) using the blastn program in BLAST+ 2.6.0 [39] with an e-value cutoff of $1 \times 10^{-5}$. Reference genomes Setaria italic v2.0, Sorghum bicolor NCBIv3, Oryza sativa Japonica Build 4.0 were downloaded from the NCBI genome database. Marker sequences that showed hits to reference genomes were used for further comparative analysis. Comparative results were visualized using the dot-plot in $\mathrm{R}$ package ggplot 2 and Circos plot in Circos package $[40,41]$.

\section{QTL mapping of turf quality-related traits}

Turf quality-related traits evaluated by Kimball et al. were used for QTL mapping [10]. All hybrids together with parental lines were planted in a randomized completed block design (RCBD) with three replications at two locations (Raleigh and Laurel Springs, NC, United States) and evaluated for two years (2013 and 2015). Turf quality-related traits, including overall turf quality, leaf texture, genetic color, and turf density were evaluated visually on a 1 to 9 scale according to the National Turfgrass Evaluation Program's (NTEP) guidelines as follows: turf quality, 1 = poor quality and $9=$ excellent quality; leaf texture, $1=$ coarsest texture and $9=$ finest texture; genetic color, $1=$ light green/yellow and $9=$ dark green; turf density, 1 = sparsest density and $9=$ densest turf. Each year by each location combination was considered as a separate environment. An analysis of variance (ANOVA) and least square (LS) means were generated using the GLM procedure in SAS statistical software version 9.4 (SAS Inst. Inc., 2017) for each trait. QTL analysis was performed using LS mean values both for individual environments and across environments against the integrated linkage map using MapQTL 6.0 [42]. Interval mapping (IM) and multiple QTL method (MQM) analysis were performed to detect significant associations between markers and phenotypic traits using a regression approach. LOD thresholds $(P<0.05)$ for genome-wide were determined for each trait using a 
permutation test with 10,000 iterations. Regions with a LOD score above threshold values were considered as potential QTL intervals. Allelic effects were estimated as $\mathrm{A}_{\mathrm{f}}=\left[\left(\mu_{\mathrm{ac}}+\mu_{\mathrm{ad}}\right)-\left(\mu_{\mathrm{bc}}+\mu_{\mathrm{bd}}\right)\right] / 4$ for female (Raleigh) additivity; $\mathrm{A}_{\mathrm{m}}=\left[\left(\mu_{\mathrm{ac}}+\mu_{\mathrm{bc}}\right)-\left(\mu_{\mathrm{ad}}+\mu_{\mathrm{bd}}\right)\right] / 4$ for male (Seville) additivity and $\mathrm{D}=\left[\left(\mu_{\mathrm{ac}}+\mu_{\mathrm{bd}}\right)-\left(\mu_{\mathrm{ad}}+\mu_{\mathrm{bc}}\right)\right] / 4$ for dominance where $\mu_{\mathrm{ac}}, \mu_{\mathrm{ad}}, \mu_{\mathrm{bc}}$ and $\mu_{\mathrm{bd}}$ are estimated phenotypic means associated to each of the 4 possible genotypic classes ac, bc, ad and bd, deriving for an $a b \times$ cd cross [43]. Furthermore, sequences of markers within the identified regions of interest were searched against the NCBI NR database using blastn/blastp tools to obtain their orthologs. Gene Ontologoy (GO) annotation was conducted using UniProt database to predict gene function in the QTL regions.

\section{Additional file}

Additional file 1: Table S1 to S12. Table S1. Sequence of barcodes and adapters in St. Augustinegrass GBS library. Table S2. Number of sequencing reads for St. Augustinegrass parent lines and hybrids. Table S3. Detail of linkage group and marker sequences of 'Raleigh' St. Augustinegrass genetic map. Table S4. Detail of linkage group and marker sequences of 'Seville' St. Augustinegrass genetic map. Table S5. Detail of linkage group and marker segregation in integrated St. Augustinegrass genetic map. Table S6. Genomics comparison between 'Raleigh' St. Augustinegrass linkage groups and foxtail millet genome. Table S7. Genomics comparison between 'Seville' St. Augustinegrass linkage groups and foxtail millet genome. Table S8. Genomics comparison between 'Raleigh' St. Augustinegrass linkage groups and sorghum genome. Table S9. Genomics comparison between 'Seville' St. Augustinegrass linkage groups and sorghum genome. Table S10. Genomics comparison between 'Raleigh' St. Augustinegrass linkage groups and rice genome. Table S11. Genomics comparison between 'Seville' St. Augustinegrass linkage groups and rice genome. Table S12. Gene ontology analysis of sequence within QTL regions related to leaf development. (XLSX 346 kb)

\section{Abbreviations}

Chr: Chromosome; cM: Centimorgan; GBS: Genotyping by sequencing; GO: Gene ontology; GWAS: Genome-wide association study; IM: Interval mapping; LG: Linkage group; LOD: Logarithm of the odds; MAS: Markerassisted selection; MQM: Multiple QTL method; NTEP: National Turfgrass Evaluation Program; QTL: Quantitative trait locus; SNP: Single nucleotide polymorphism; SSR: Simple sequence repeat

\section{Acknowledgements}

The authors would like to thank the NC State University Genomic Science Laboratory (Raleigh, NC, USA) for the next generation sequencing service and Jared Smith with the USDA-ARS Eastern Regional Small Grains Genotyping Lab (ERSGGL) (Raleigh, NC, USA) for help with GBS library construction.

\section{Funding}

This research was supported in part by funding provided by the North Carolina Crop Improvement Association, the North Carolina State University Center for Turfgrass Environmental Research and Education, and by the Specialty Crop Research Initiative [2015-51181-24291] from the USDA National Institute of Food and Agriculture. Authors declare that none of the funding bodies have any role in the design of the study and collection, analysis, and interpretation of data as well as in writing the manuscript.

\section{Availability of data and materials}

All the raw sequencing data was deposited into the database of the sequence read archive (SRA) in the National Center for Biotechnology Information (NCBI) under SRA ID SRP149564. Other datasets used during the current study are available from the corresponding author upon reasonable request.

\section{Authors' contributions}

$\mathrm{XY}$ was responsible for designing and performing the experiments, data analysis and manuscript writing. JK performed the phenotypic evaluations. SML conceived the project, maintained the population, provided feedback on the proposed work and revised the manuscript before submission. All authors read, edited, and approved the final manuscript.

\section{Ethics approval and consent to participate}

Not applicable.

\section{Consent for publication}

Not applicable.

\section{Competing interests}

The authors declare that they have no competing interests.

\section{Publisher's Note}

Springer Nature remains neutral with regard to jurisdictional claims in published maps and institutional affiliations.

\section{Author details}

'Department of Crop and Soil Sciences, N.C. State University, Box 7620, Raleigh, NC 27695-7620, USA. ²Department of Agronomy and Plant Genetics, University of Minnesota, St. Paul, MN 55108-6026, USA.

Received: 5 June 2018 Accepted: 21 November 2018

Published online: 12 December 2018

\section{References}

1. Sauer JD. Revision of Stenotaphrum (Gramineae: Paniceae) with attention to its historical geography. Brittonia. 1972;24:202-22.

2. Busey P, Davis EH. Turfgrass in the shade environment. Proc Fla State Hort Soc. 1991;104:353-8.

3. Milla-Lewis SR, Zuleta MC, Van Esbroeck GA, Quesenberry KH, Kenworthy KE. Cytological and molecular characterization of genetic diversity in Stenotaphrum. Crop Sci. 2013;53(1):296-308.

4. Kellogg EA. Flowering Plants. Monocots: Poaceae (Vol. 13). New York: Springer; 2015.

5. Parrish DJ, Fike JH. The biology and agronomy of switchgrass for biofuels. Crit Rev Plant Sci. 2005;24(5-6):423-59.

6. Giussani LM, Cota-Sánchez JH, Zuloaga FO, Kellogg EA. A molecular phylogeny of the grass subfamily Panicoideae (Poaceae) shows multiple origins of C4 photosynthesis. Am J Bot. 2001;88(11):1993-2012.

7. Long JA, Bashaw EC. Microsporogenesis and chromosome numbers in St. Augustinegrass. Crop Sci. 1961;1:41-3.

8. Mulkey SE. SSR development and mapping of gray leaf spot resistance genes in St. Augustinegrass (Master's thesis, North Carolina State University, Raleigh). 2012. Retrieved from http://www.lib.ncsu.edu/resolver/1840.16/ 8590/.

9. Mulkey SE, Zuleta MC, Keebler JE, Schaff JE. Milla-Lewis SRDevelopment and characterization of simple sequence repeat markers for St. Augustinegrass Crop Sci. 2014;54:401-12.

10. Kimball JA, Tuong TD, Arellano C, Livingston DP III, Milla-Lewis SR. Linkage analysis and identification of quantitative trait loci associated with freeze tolerance and turf quality traits in St. Augustinegrass. Mol Breeding. 2018;38:67.

11. Cronn R, Liston A, Parks M, Gernandt DS, Shen R, Mockler T. Multiplex sequencing of plant chloroplast genomes using Solexa sequencing-bysynthesis technology. Nucleic Acids Res. 2008;36:e122.

12. Huang YF, Poland JA, Wight CP, Jackson EW, Tinker NA. Using genotypingby-sequencing (GBS) for genomic discovery in cultivated oat. PLoS One. 2014;9:e102448.

13. Elshire RJ, Glaubitz JC, Sun Q, Poland JA, Kawamoto K, Buckler ES, et al. A robust, simple genotyping-by-sequencing (GBS) approach for high diversity species. PLoS One. 2011;6:e19379.

14. Beissinger TM, Hirsch CN, Sekhon RS, Foerster JM, Johnson JM, Muttoni G, et al. Marker density and read depth for genotyping populations using genotyping-by-sequencing. Genetics. 2013;193:1073-81. 
15. Lu F, Lipka AE, Glaubitz J, Elshire R, Cherney JH, Casler MD, Buckler ES, Costich DE. Switchgrass genomic diversity, ploidy, and evolution: novel insights from a network-based SNP discovery protocol. PLoS Genet. 2013; 9(1):e1003215.

16. Sonah H, Bastien M, Iquira E, Tardivel A, Legare G, Boyle B, et al. An improved genotyping by sequencing (GBS) approach offering increased versatility and efficiency of SNP discovery and genotyping. PLoS One. 2013:8:e54603.

17. Holloway HM, Yu XW, Dunne JC, Schwartz BM, Patton AJ, Arellano C, et al. A SNP- based high-density linkage map of zoysiagrass (Zoysia japonica Steud.) and its use for the identification of QTL associated with winter hardiness. Mol Breeding. 2018;38:10.

18. Rouppe van der Voort J, van Eck H, van Zandvoort P, Overmars H, Helder J, Bakker J. Linkage analysis by genotyping of sibling populations: a genetic map for the potato cyst nematode constructed using a "pseudo-F2" mapping strategy. Mol Gen Genet. 1999;261:1021-31.

19. Van Ooijen JW. JoinMap 4, Software for the calculation of genetic linkage maps in experimental populations. Wageningen: Kyazma BV; 2006.

20. Bonos SA, Clarke BB, Meyer WA. Breeding for disease resistance in the major cool-season turfgrasses. Annu Rev Phytopathol. 2006;44:213-34.

21. Wang F, Singh R, Genovesi AD, Wai CM, Huang X, Chandra A, Yu Q. Sequence-tagged high-density genetic maps of Zoysia japonica provide insights into genome evolution in Chloridoideae. Plant J. 2015;82(5):744-57.

22. Wu J, Li LT, Li M, Khan MA, Li XG, Chen H, et al. High density genetic linkage map construction and identification of fruit-related QTLs in pear using SNP and SSR markers. J Exp Bot. 2014;65:5771-81.

23. Wu QH, Chen YX, Zhou SH, Fu L, Chen JJ, Xiao Y, et al. High-density genetic linkage map construction and QTL mapping of grain shape and size in the wheat population Yanda1817 $\times$ Beinong6. PLoS One. 2015;10:e0118144.

24. Srinivasachary DMM, Gale MD, Devos KM. Comparative analyses reveal high levels of conserved colinearity between the finger millet and rice genomes. Theor Appl Genet. 2007;115(4):489-99.

25. Luo MC, Deal KR, Akhunov ED, Akhunova AR, Anderson OD, Anderson JA et al. Genome comparisons reveal a dominant mechanism of chromosome number reduction in grasses and accelerated genome evolution in Triticeae. P Natl Acad Sci USA. 2009;106:15780-5.

26. Wang $X$, Jin $D$, Wang $Z$, Guo $H$, Zhang L, Wang L, Li J, Paterson AH. Telomere-centric genome repatterning determines recurring chromosome number reductions during the evolution of eukaryotes. New Phytol. 2015; 205:378-89.

27. Salse J, Bolot S, Throude M, Jouffe V, Piegu B, Quraishi UM, Calcagno T, Cooke R, Delseny M, Feuillet C. Identification and characterization of shared duplications between rice and wheat provide new insight into grass genome evolution. Plant Cell. 2008;20:11-24.

28. Thiel T, Graner A, Waugh R, Grosse I, Close TJ, Stein N. Evidence and evolutionary analysis of ancient whole-genome duplication in barley predating the divergence from rice. BMC Evol Biol. 2009;9: 209-26.

29. Devos KM. Grass genome organization and evolution. Curr Opin Plant Biol. 2010;13:139-45.

30. Zhang G, Liu X, Quan Z, Cheng S, Xu X, Pan S, et al. Genome sequence of foxtail millet (Setaria italica) provides insights into grass evolution and biofuel potential. Nat Biotechnol. 2012;30:549-54.

31. Cavagnaro PF, Iorizzo M, Yildiz M, Senalik D, Parsons J, Ellison S, Simon PW. A gene-derived SNP-based high resolution linkage map of carrot including the location of QTL conditioning root and leaf anthocyanin pigmentation. BMC Genomics. 2014;16(15):1118.

32. Lynn K, Fernandez A, Aida M, Sedbrook J, Tasaka M, Masson P, et al. The PINHEAD/ZWILLE gene acts pleiotropically in Arabidopsis development and has overlapping functions with the ARGONAUTE1 gene. Development. 1999; 126(3):469-81.

33. Nishimura A, Ito M, Kamiya N, Sato $Y$, Matsuoka M. OsPNH1 regulates leaf development and maintenance of the shoot apical meristem in rice. Plant J. 2002;30(2):189-201.

34. Li R, Li J, Li S, Qin G, Novák O, Pěnčík A, et al. ADP1 affects plant architecture by regulating local auxin biosynthesis. PLoS Genet. 2014; 10(1):e1003954

35. Zhang H, Zhu H, Pan Y, Yu Y, Luan S, Li L. A DTX/MATE-type transporter facilitates abscisic acid efflux and modulates ABA sensitivity and drought tolerance in Arabidopsis. Mol Plant. 2014;7(10):1522-32.
36. Poland J, Brown PJ, Sorrells ME, Jannink J. Development of high-diversity genetic maps for barley and wheat using a novel two-enzyme genotypingby sequencing approach. PLoS One. 2012;7(2):e32253.

37. Glaubitz JC, Casstevens TM, Lu F, Harriman J, Elshire RJ, Sun Q, Buckler ES. TASSEL-GBS: a high capacity genotyping by sequencing analysis pipeline. PLoS One. 2014;9:e90346.

38. Voorrips RE. MapChart: software for the graphical presentation of linkage maps and QTLs. J Hered. 2002;93(1):77-8.

39. Camacho C, Coulouris G, Avagyan V, Ma N, Papadopoulos J, Bealer K, Madden TL. BLAST+: architecture and applications. BMC Bioinformatics. 2009;10:421

40. Wickham H. ggplot2: elegant graphics for data analysis. New York: SpringerVerlag; 2016. ISBN 978-3-319-24277-4. http://ggplot2.org/.

41. Krzywinski M, Schein J, Birol I, Connors J, Gascoyne R, Horsman D, Jones SJ, Marra MA. Circos: an information aesthetic for comparative genomics. Genome Res. 2009;19:1639-45.

42. Van Ooijen JW. MapQTL 6: software for the mapping of quantitative trait loci in experimental populations of diploid species. Wageningen: Kyazma BV; 2009.

43. Ben Sadok I, Celton JM, Essalouh L, Zine El Aabidine A, Garcia G, Martinez S, et al. QTL mapping of flowering and fruiting traits in olive. PLoS One. 2013:8:e62831.
Ready to submit your research? Choose BMC and benefit from:

- fast, convenient online submission

- thorough peer review by experienced researchers in your field

- rapid publication on acceptance

- support for research data, including large and complex data types

- gold Open Access which fosters wider collaboration and increased citations

- maximum visibility for your research: over $100 \mathrm{M}$ website views per year

At BMC, research is always in progress.

Learn more biomedcentral.com/submissions 